\title{
Analog to Digital Converters (ADC): A Literature Review
}

\author{
*Hemlata Dalmia ${ }^{1}$ and Sanjeet K. Sinha ${ }^{2}$ \\ ${ }^{1}$ School of Electronics and Electrical Engineering, Lovely Professional University, Punjab, India. \\ ${ }^{2}$ School of Electronics and Electrical Engineering, Lovely Professional University, Punjab, India.
}

\begin{abstract}
The signal processing is advancing day by day as its needs and in wireline/wireless communication technology from $2 \mathrm{G}$ to $4 \mathrm{G}$ cellular communication technology with CMOS scaling process. In this context the high-performance ADCs, analog to digital converters have snatched the attention in the field of digital signal processing. The primary emphasis is on low power approaches to circuits, algorithms and architectures that apply to wireless systems. Different techniques are used for reducing power consumption by using low power supply, reduced threshold voltage, scaling of transistors, etc. In this paper, we have discussed the different types and different techniques used for analog to digital conversion of signals considering several parameters.
\end{abstract}

\section{Introduction}

In digital processing systems, analog to digital converter (ADC) is a basal unit playing an imperative role as digital processing overweighs the analog processing in most of the applications. ADC bridges the analog trend with digital reign. For high demands, the ADC speed and power consumption are being made major concerns. Successive approximate register (SAR) ADC is acknowledged in many ADC applications for its efficacy in power and high competence in speed $[1,2]$.

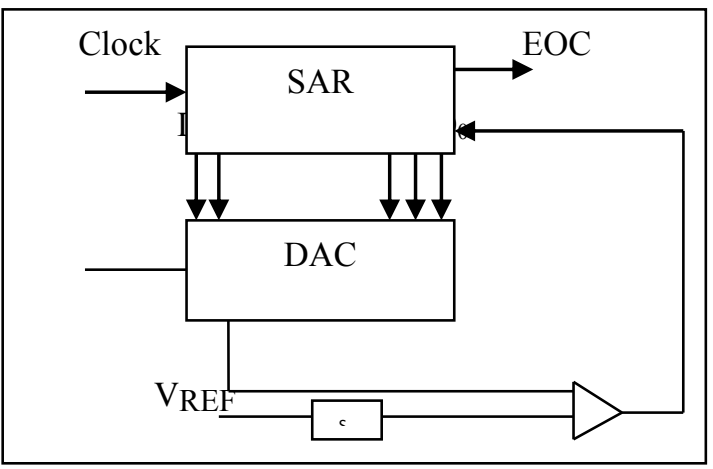

Fig. 1: Block Diagram of SAR ADC

The works mentioned in this paper employed different approaches to characterize ADC. In some approaches, quasi-static measurements like differential non linearity (DNL) and integral nonlinearity (INL) are measured and *Hemlata Dalmia, dalmiahemlata@gmail.com some approaches measure dynamic parameters like SNDR (signal to noise distortion ratio) and SFDR (spurious free dynamic range) using different tools The ADC in successive approximate register type works basically as illustrated in the block diagram of fig. 1 . Analog to digital converters have many applications ranging from sensors, audio and DAS to video, radar and communication interfaces. In recent demands towards high-speed serial links where ADC is demanded of high speed and low power dissipation.

\section{Review of Literature}

The author M Sani and A Anasi Hamoui proposed an implementation of digital background calibration technique in 13-bit pipelined ADC and behavioral simulation of the same using SIMULINK, keeping $\sigma=$ $0.25 \%$ of capacitor mismatches, the SNR value improvement is from 10 to 12.5 bits and SFDR of $95 \mathrm{~dB}$ [3].

In 2008, Imran Ahmed presented a technique to calibrate 11- bit pipelined ADC for improving SNDR from $46.9 \mathrm{~dB}$ to $60 \mathrm{~dB}$ and SFDR from $48.9 \mathrm{~dB}$ to 70 $\mathrm{dB}$ using fabrication prototyped on $0.18 \mu \mathrm{m}$ process and the calibration was done in the backend using the clock cycle of $10^{4}$ [4]. In 2009, the authors proposed the concept of split calibration technique in 12- bits ADC to improve SFDR and SNDR using SIMULINK. In this article, the error in gain had been considered along with capacitor mismatch. It was showed by behavioural simulation the increase in SNDR and SFDR values from 
56.4 to $73.8 \mathrm{~dB}$ and the whole calibration process was performed in approximately $10^{5}$ cycles [5].

The authors 'A Panigada and I Galton" have proposed two techniques for background calibration to counteract two problems of gain error and capacitor mismatch in pipelined ADC ie HDC (harmonics distortion correction) and DNC (DAC noise cancellation). Using HDC algorithm for gain error correction, implementation has achieved SNDR of $70 \mathrm{~dB}$ and SFDR of $85 \mathrm{~dB}$ in a $90 \mathrm{~nm}$ CMOS process with power consumption of $130 \mathrm{~mW}$ [6]. In 2011, Lee and Flynn worked with SAR ADC which achieved high energy efficiency with good resolution and high-speed operation resulting in low power using calibration free technique. The fabrication in $65 \mathrm{~nm}$ as well as in $90 \mathrm{~nm}$ CMOS was utilised to get the results of speed of $50 \mathrm{MS} / \mathrm{s}$ and efficient number of bits of $10.4 \mathrm{~b}$ at Nyquist [7].

The author presented a 10 bits pipelined ADC in which switching opamps and digital calibration techniques are employed to achieve low power and low error gain using $65 \mathrm{~nm}$ CMOS technology for fabrication process. In this article, SNDR and SFDR values obtained are $55.4 \mathrm{~dB}$ and $67.2 \mathrm{~dB}$ from a supply of $1 \mathrm{~V}$ with low power consumption of $26.6 \mathrm{~mW}$ and chip area of $0.36 \mathrm{~mm}^{2}$ [8]. In one article of 2014 , a prototyped time interleaved SAR ADC fabricated on $65 \mathrm{~nm}$ CMOS technology with high enhanced speed by Flash ADC among eight SAR ADCs was proposed. In this paper an approach of considering Flash ADC as reference for timing skew calibration technique to be used [9], clearly shown in the fig 2 .

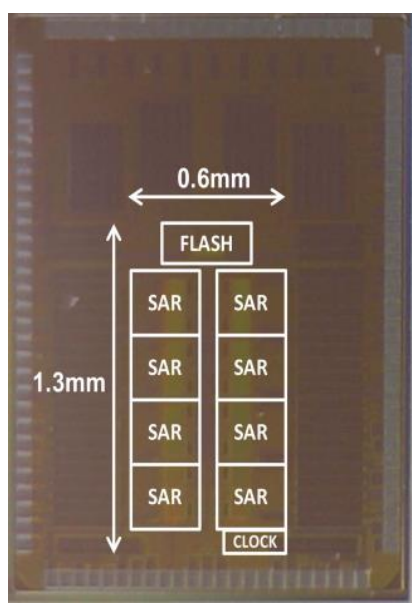

Fig. 2.: Die photograph of Time Interleaved ADC [9]

For high resolution SAR ADCs, a coarse reference ADC acceleration technique is employed in a new background calibration technique to improve convergence speed and ADCs linearity performance. The calibration is performed on the basis of IRD technique (internal redundancy dithering) [10].

The authors in 2015 employed a fully deterministic approach for digital calibration based on split 12-bit pipeline $\mathrm{ADC}$ at multistage level to find the circuit errors. The calibration was performed on a $200 \mathrm{MS} / \mathrm{s} 40$ $\mathrm{nm}$ CMOS for the modification in capacitor mismatch and amplifier gain. In this article, the behavioural simulation proved the area and power of the implemented 40nm 12-bit pipelined ADC $0.42 \mathrm{~mm}^{2}$ and $54 \mathrm{~mW}[11]$.

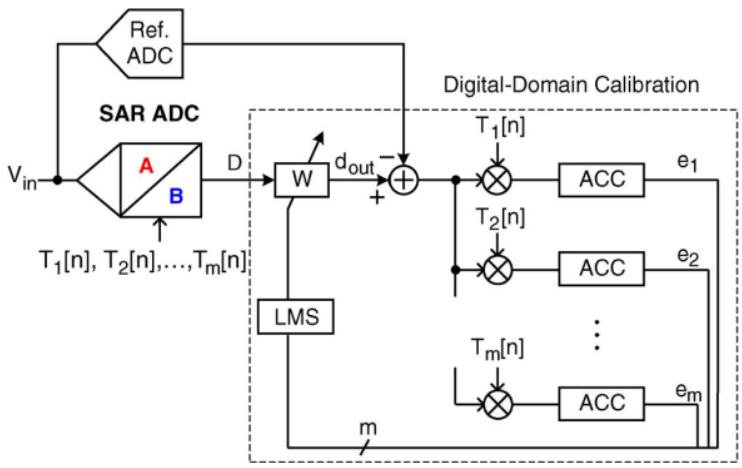

Fig. 3: IRD approach extended to treat $m$ significant bits in SAR ADC. Also shown is a reference ADC that removes the input signal before bit-weight correlation takes place [10].

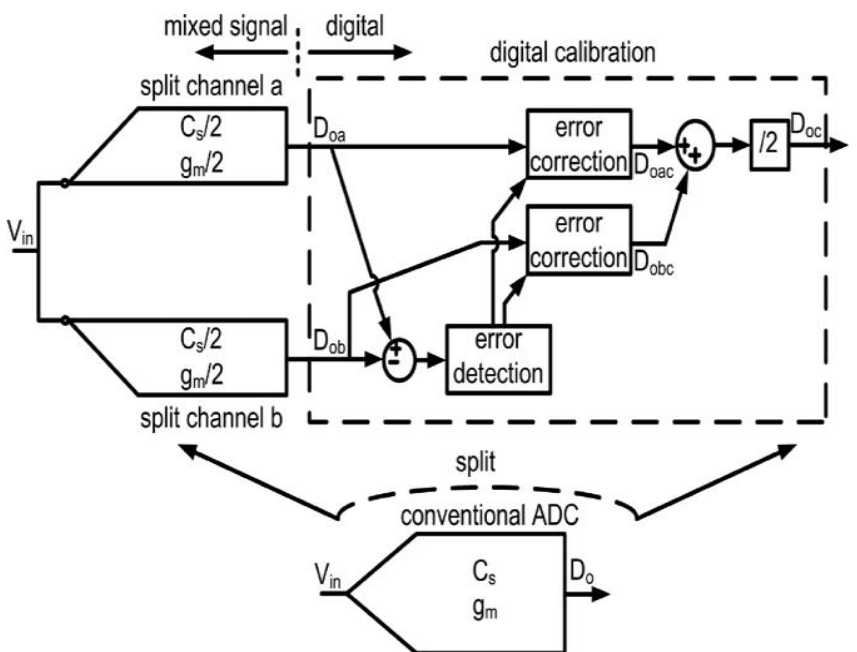

Fig 4: Split ADC background calibration concept [11]

Another work by A. Fahmy et al. on stochastic ADC was explained with its programmability and reconfigurability by dividing the whole design into 8 channels with a 10-bit control word. Using Verilog and digital design tools for synthesis fabricated on $130 \mathrm{~nm}$ CMOS process, results were obtained in terms of SFDR and SNDR at $0.7 \mathrm{~V}$ supply [12]. Another publication introduced and verified another technique of LMS algorithm (least mean squares) for background calibration for pipelined ADCs to correct conversion 
errors and DRDE (digitized residue distance estimation) algorithm [13]. The authors M.A. Montazerolghaem et. Al propose a digital background calibration technique with LMS algorithm to righteous the capacitor mismatch and error gains in which the conversion time is significantly reduced as compared to the same LMS techniques used in the other publication in the year 2014 by $\mathrm{B}$. Zenali. The technique was simulated on 12-bit $100 \mathrm{MS} / \mathrm{s}$ spilt pipelined ADC [14]. Another work attained shorter calibration times for split pipelined ADC and handled the non-orthogonality's of calibration loop. Using the calibration technique, the power dissipation in the residue amplifier was proved to be 60 $\%$ efficient and was estimated to be $192 \mathrm{~mW}$ with the SFDR and SNDR improvements [15].

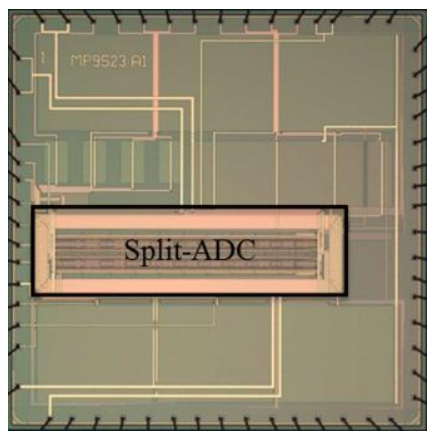

Fig 5: Chip micrograph of Split ADC [15]

In 2003, high speed 12-bits pipe-lined ADCs is presented for low power digital signal processing system in which the precision amplifiers are employed to cancel nonlinearities due to efficient simple power open-loop stages[16].In this, power reduction is done by the cancellation of non- linearities [16].

In this, the author has introduced a "split ADC" architecture running continuous digital background calibration technique in which the both ADCs used converts the same signal and after calibration, the outputs of both ADCs must be same and their difference produces null. In this, the algorithmic converter is realized as spilt ADC with 1000 conversions in self calibration. This consumes power of 1.5 milliwatts having die size of $1.2 \mathrm{~mm}$ X $1.4 \mathrm{~mm}$ [17]. The authors L. Dorrer \& F. Kuttner in 2005 explained that the low power is achieved by using three comparators in tracking ADC. The loop stability was increased and improved and also reduced the loop delay time. It is proven that the ADC is consuming power of 3 milliwatts with $1.5-\mathrm{V}$ supply at clock frequency of $104 \mathrm{MHz}$. It is also proven that this design is consuming less power than that of the conventional start-of-the-art continuous time [18].

Also, A Zandieh et, al. presented a TI-ADC (timeinterleaved Analog to digital converter) achieving more *Hemlata Dalmia, dalmiahemlata@gmail.com bandwidth and highest sampling rate with a 5-bit resolution using a comparator of low power and latch along with track and hold amplifiers [19].

In this, the author has presented a 12-bit pipelined ADC and proposed a digital background calibration technology to improve its performance in cancelling the errors like capacitor mismatch, errors in gain and nonlinearities produced, on 90nm CMOS technology [20].

One more related article proposing SAR assisted digital slope ADC, had brought the leads of both SAR and digital slope ADCs in a hybrid ADC. The prototype of hybrid ADCs was fabricated on $28 \mathrm{~nm}$ CMOS technology resulting into very less power of $0.35 \mathrm{~mW}$ and occupying less area of $66 \mu \mathrm{m} \times 71 \mu \mathrm{m}$ [21], the micrograph chip of the same prototype is shown in fig 6 .

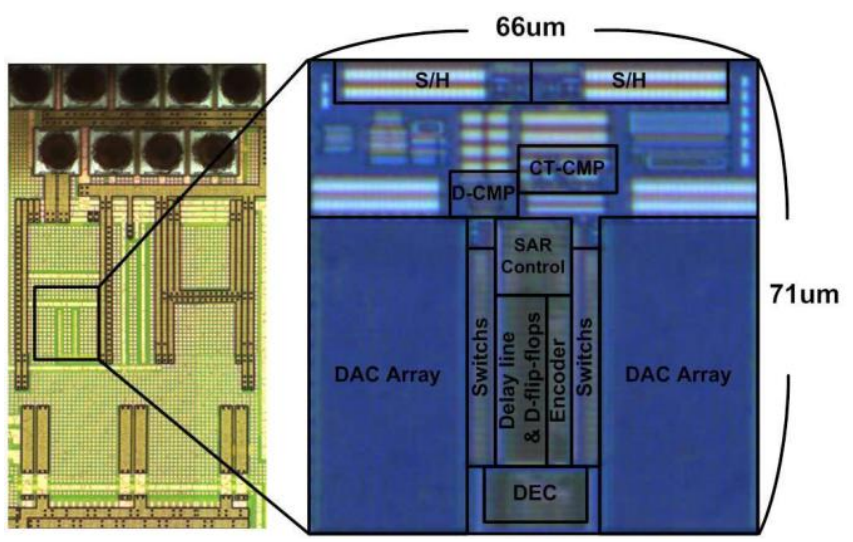

Fig 6: zoomed in view of ADC core [21]

A flash ADC is presented to improve chip area and performance speed with high resolution by employing multi segment encoder with MOS current mode logic (MCML) in the article [22]. In this article the novel structure maintains the simplicity of the encoder structure by using two segment encoders and a smaller number of multiplexers [22]. Another discussion on case study of pipelined ADC presented in the same year in which a vigorous calibration technique of multivalued ADC using the integral INL based calibration is obtained [23]. The paper [24] presented extended counting ADC without calibration to be applied in CMOS image sensors. In the same year a digitally assisted offset calibration technique is proposed by the authors for high speed open loop residual amplifier to achieve correct operation of 10-bit ADC and SNDR pf $22.5 \mathrm{~dB}$ and SFDR of $11 \mathrm{~dB}$ for 13 -bit pipeline ADC [25].

In this, the power consumption was lowered by using a tracking ADC in which three comparators are exercised for wireless applications. The loop stability was increased and improved and also reduced the loop delay time. It is proven that; the ADC is consuming $3 \mathrm{~mW}$ from a $1.5-\mathrm{V}$ supply when it is clocked at $104 \mathrm{MHz}$. it is also proven that this design is consuming less power 
than that of the conventional start-of-the-art continuous time [26]. In this, simple power efficient open-loop stages are replaced in the place of precision amplifiers as precision amplifiers dominate the power dissipation in most high-speed pipe-lined ADCs. In this, power reduction is done by combining digital domain estimation and cancellation of non-linearities with simple power efficient open-loop stages. This can be used for efficient low power digital signal processing [27].

KB Vaibhav et al., presented a review article for high speed ADCs with comparison of different architectures with low power CMOS. The article details Flash ADCs, pipelined ADCs, SAR ADCs [28-30], subranging and two step ADCs, interpolating and folding ADCs to be suitable for the hybrid ADC design to perform high speed operations with the benefit in power values. The paper also talks about the hybrid ADC for applications demanding 1-GS/s with 6-bits resolution [31].

In 2019, the author Savitha et, al. has proposed a SARADC with dual split 3-sections capacitive array DAC for IoT systems to obtain good accuracy for peer communication functioning like 14-bit SAR ADC [32] for low cost CMOS development. The authors $\mathrm{T}$ Hung et, al. proposed split ADC with digital background calibration for the improvement in the gain and INL errors in a pipelined ADC by employing 12-bit 400MS/s pipelined ADC [33]. The presented ADC in [34] used a slew rate boosting technique with Class $\mathrm{C}$ for saving power in the residual amplifiers of 12-bit architecture giving good slew rate performance by the author Mohd Naved et, al. SAR ADC are researched and described for different applications such as Biomedical applications, wireless sensors, receivers, etc. [35,36,37,39,40,42] for its high speed performance. Another type of ADC: Flash ADC $[38,41,43]$ is also proposed for many applications such as high-speed instrumentation, radar, wireless sensor network, DSO, digital TVs and so on.

Some of the articles discussed and reviewed the various types of ADC and different calibration methods for timing skews in ADCs [44-51]. The SAR ADC [52-56] with pipelined $\mathrm{ADC}[57,58]$ combination is popularly in trend to get good performance from ADC along with CMOS scaling [59]. The reconfigurable ADC [60-64] are in research using pipelined and SAR ADCs to configure the resolution and sampling rate depending upon the requirements.

SAR ADC [68-72] is an analog circuit whose performance gets better with CMOS scaling process [73-
75] and always employed for high speed, low power and high-resolution ADCs. The publication history of SAR $\mathrm{ADC}$ is noticeable from last two decades studying its performance enhancement [76-77].

In fig 7, the survey is plotted on the published articles based on SAR ADC till 2019 in VLIS and till 2020 in ISSCC, taken from the survey [78].

The prevailing research proves that the hybrid ADCs [65-67] architecture is more advantageous for enhancement in terms of speed and for low power operations [68]. The hybrid ADCs [79-82] have different combinations and blocks as per the applications demand. The designers can obtain better performances of ADC than in a monolithic chip with the help of Hybrid ADCs [83-90]. Employing Hybrid structured ADC [91-94], different parameters of ADC such as sampling rate, scaling, resolution, calibration free and so on can be improved.

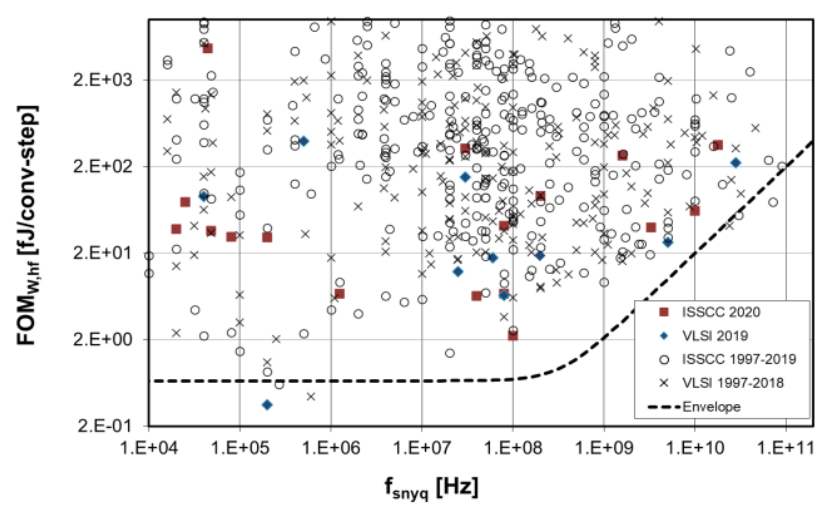

Fig 7: SAR ADC published till 2020 in ISSC and till 2019 in VLSI [78]

\section{Results and Discussions}

The Hybrid ADC comprising of pipelined SAR ADC with Digital Amplifiers concept was proposed, designed and implemented in $28 \mathrm{~nm}$ CMOS achieving high speed of $320 \mathrm{MS} / \mathrm{s}$ by observing on supply of both $0.7 \mathrm{~V}$ and $0.9 \mathrm{~V}$, with SNDR of $60 \mathrm{~dB}$ [69]. The result FFT simulations of the pipelined SAR ADC were imported to MATLAB, for post simulation tests shown in figure 8 . The proposal is to design a calibration free and CMOS scalable Hybrid ADC with digital amplifier instead of conventional OPAMPs. 


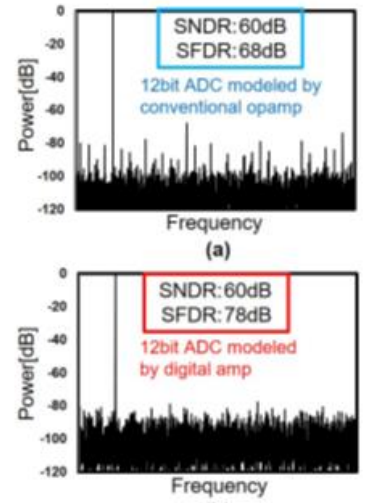

(b)
Fig. 8: FFT results of pipelined SAR ADC simulated on MATLAB with both conventional amplifiers and Digital Amplifiers [69].

\section{Conclusion}

The detailed survey is done on different types and various techniques of ADC and the summary of various features is presented using respective headings. Considering the merits and demerits of each techniques one should select the appropriate techniques for the end application. The analysis and trend prove the frequent employed of SAR ADC due its highly efficient with high speed performance and prominent scaling process. The features and limitations of various techniques are summarized and compared. The survey proposes to use Hybrid ADC using SAR pipelined ADC.

TABLE I. Comparison between various ADCs based on different parameters

\begin{tabular}{|c|c|c|c|c|c|c|c|}
\hline $\begin{array}{c}\text { Reference } \\
\text { No. }\end{array}$ & $\begin{array}{c}\text { Technology } \\
(\mu \mathrm{m})\end{array}$ & $\begin{array}{l}\text { Sampling } \\
\text { rate }(S / s)\end{array}$ & $\begin{array}{c}\text { Power } \\
\text { consumption } \\
\text { (W) }\end{array}$ & $\begin{array}{c}\text { Supply } \\
\text { voltage }(V)\end{array}$ & $\begin{array}{c}\text { FOM } \\
\text { (J/conversion } \\
\text { step) }\end{array}$ & $\begin{array}{c}\text { SNDR } \\
\text { (dB) }\end{array}$ & Resolution \\
\hline 4 & 0.18 & $45 \mathrm{MS} / \mathrm{s}$ & - & 1.8 & - & $70 \mathrm{~dB}$ & \\
\hline 6 & 0.09 & - & $130 \mathrm{~mW}$ & - & - & $70 \mathrm{~dB}$ & - \\
\hline 12 & 0.13 & $100 \mathrm{MS} / \mathrm{s}$ & $1.5 \mathrm{~mW}$ & $0.7 \mathrm{~V}$ & - & $45 \mathrm{~dB}$ & 10 bits \\
\hline 18 & 0.13 & - & $3 \mathrm{~mW}$ & $1.5 \mathrm{~V}$ & - & - & 4 bits \\
\hline 17 & 0.25 & $1 \mathrm{MS} / \mathrm{s}$ & $105 \mathrm{~mW}$ & $2.5 \mathrm{~V}$ & - & - & 16-bits \\
\hline 21 & 0.028 & $100 \mathrm{MS} / \mathrm{s}$ & $0.35 \mathrm{~mW}$ & $0.9 \mathrm{~V}$ & 2.6 & $64.43 \mathrm{~dB}$ & - \\
\hline 35 & 0.13 & $1.6-\mathrm{GS} / \mathrm{s}$ & $163 \mathrm{~m}$ & 1.2 & $4.08 \mathrm{p}$ & $29.7 \mathrm{~dB}$ & - \\
\hline 36 & 0.065 & $1 \mathrm{GS} / \mathrm{s}$ & $31.5 \mathrm{~mW}$ & 1.2 & 20.9 fJ/step & $64.26 \mathrm{~dB}$ & 12-bits \\
\hline 37 & 0.18 & $5 \mathrm{MS} / \mathrm{s}$ & $8 \mathrm{nW}$ & $1.8 \mathrm{~V}$ & $20 \mathrm{fJ} /$ conv. & - & - \\
\hline 38 & 0.065 & $900 \mathrm{MS} / \mathrm{s}$ & $3.5 \mathrm{~mW}$ & $1 \mathrm{~V}$ & $32 \mathrm{fJ} /$ conv.-step & $49.5 \mathrm{~dB}$ & 8-bits \\
\hline 39 & - & $1 \mathrm{MS} / \mathrm{s}$ & - & - & - & $75.5 \mathrm{~dB}$ & 14-bits \\
\hline 40 & 0.18 & - & $1 \mathrm{~mW} /$ element & $1.8 \mathrm{~V}$ & - & 51.8 & - \\
\hline 42 & 0.18 & $178.6 \mathrm{KS} / \mathrm{s}$ & $8 \mu \mathrm{W}$ & $1.8 \mathrm{~V}$ & - & $37 \mathrm{~dB}$ & 6-bits \\
\hline 52 & 0.045 & - & $330.5 \mu \mathrm{W}$ & - & - & $33.915 \mathrm{~dB}$ & 10 bits \\
\hline 53 & 0.13 & $1 \mathrm{GS} / \mathrm{s}$ & $26 \mathrm{~mW}$ & $1.2 \mathrm{~V}$ & & $49.7 \mathrm{~dB}$ & 6-bits \\
\hline 54 & $0.18 \mu \mathrm{m}$ & $10 \mathrm{GS} / \mathrm{s}$ & $1.5 \mathrm{~mW}$ & $1 \mathrm{~V}$ & - & - & 6-bits \\
\hline 70 & 0.04 & $6.14 \mathrm{MS} / \mathrm{s}$ & $6.43 \mu \mathrm{W}$ & $0.5 \mathrm{~V}$ & 7.1 & 44.3 & - \\
\hline
\end{tabular}

\section{References:}

1. Fu, Tao, "High-Speed Successive Approximation Register (SAR) ADC Design with Multiple Concurrent Comparators" (2019). Electrical Engineering Theses and Dissertations. 28.

2. Seyed Alireza, Zahrai Seyed, Alireza Zahrai, Marvin Onabajo: Review of Analog-To-Digital Conversion Characteristics and Design Considerations for the Creation of Power-Efficient Hybrid Data Converters, (April 2018), Journal of Low Power Electronics and Applications, DOI: 10.3390/jlpea8020012

3. M. Taherzadeh-Sani and A. A. Hamoui, "Digital Background Calibration of Capacitor-Mismatch Errors in Pipelined ADCs," in IEEE Transactions on Circuits and
Systems II: Express Briefs, vol. 53, no. 9, pp. 966-970, (Sept. 2006) doi: 10.1109/TCSII.2006.879097

4. Ahmed and D. A. Johns, "An 11-Bit 45 MS/s Pipelined ADC With Rapid Calibration of DAC Errors in a Multibit Pipeline Stage," in IEEE Journal of Solid-State Circuits, vol. 43, no. 7 , pp. 1626-1637, (July 2008) doi: 10.1109/JSSC.2008.923724

5. L. Hung and T. Lee, "A Split-Based Digital Background Calibration Technique in Pipelined ADCs," in IEEE Transactions on Circuits and Systems II: Express Briefs, vol. 56, no. 11, pp. 855-859, (Nov. 2009). doi: 10.1109/TCSII.2009.2034077

6. A. Panigada and I. Galton, "A $130 \mathrm{~mW} 100 \mathrm{MS} / \mathrm{s}$ pipelined ADC with $69 \mathrm{~dB}$ SNDR enabled by digital harmonic distortion correction," 2009 IEEE International Solid-State Circuits Conference - Digest of Technical Papers, San Francisco, CA, (2009), pp. 162-163,163a.

*Hemlata Dalmia, dalmiahemlata@gmail.com 
7. C. C. Lee and M. P. Flynn, "A SAR-Assisted Two-Stage Pipeline ADC," in IEEE Journal of Solid-State Circuits, vol. 46, no. 4, pp. 859-869, (April 2011). doi: 10.1109/JSSC.2011.2108133

8. B. Fang and J. Wu, "A 10-Bit 300-MS/s Pipelined ADC With Digital Calibration and Digital Bias Generation," in IEEE Journal of Solid-State Circuits, vol. 48, no. 3, pp. 670-683, (March 20131). doi: 10.1109/JSSC.2012.2233332

9. S. Lee, A. P. Chandrakasan and H. Lee, "A $1 \mathrm{GS} / \mathrm{s} 10 \mathrm{~b}$ $18.9 \mathrm{~mW}$ Time-Interleaved SAR ADC with Background Timing Skew Calibration," in IEEE Journal of Solid-State Circuits, vol. 49, no. 12, pp. 2846-2856, (Dec. 2014). doi: 10.1109/JSSC.2014.2362851

10. G. Wang, F. Kacani and Y. Chiu, "IRD Digital Background Calibration of SAR ADC with Coarse Reference ADC Acceleration," in IEEE Transactions on Circuits and Systems II: Express Briefs, vol. 61, no. 1, pp. 11-15, (Jan. 2014). doi: 10.1109/TCSII.2013.2291051

11. H. Adel, M. Sabut and M. Louerat, "Split ADC Based Fully Deterministic Multistage Calibration for High Speed Pipeline ADCs," in IEEE Transactions on Circuits and Systems I: Regular Papers, vol. 62, no. 6, pp. 1481-1488, (June 2015). doi: 10.1109/TCSI.2015.2416813

12. A. Fahmy, J. Liu, T. Kim and N. Maghari, "An All-Digital Scalable and Reconfigurable Wide-Input Range Stochastic ADC Using Only Standard Cells," in IEEE Transactions on Circuits and Systems II: Express Briefs, vol. 62, no. 8, pp. 731-735, (Aug. 2015). doi: 10.1109/TCSII.2015.2415231

13. Hamidreza Mafia, Reza Mohammadib Hossein and Shamsib, "A statistics-based digital background calibration technique for pipelined ADCs Author links open overlay panel”, Tehran, Iran Received 3 September 2014, revised 23 May 2015, Accepted 26 July 2015, Available online 3 August (2015), published by Elsevier

14. M. A. Montazerolghaem, T. Moosazadeh and M. Yavari, "A Predetermined LMS Digital Background Calibration Technique for Pipelined ADCs," in IEEE Transactions on Circuits and Systems II: Express Briefs, vol. 62, no. 9, pp. 841-845, Sept. 2015. doi: 10.1109/TCSII.2015.2435071

15. R. Sehgal, F. van der Goes and K. Bult, "A 12 b $53 \mathrm{~mW}$ 195 MS/s Pipeline ADC with 82 dB SFDR Using SplitADC Calibration," in IEEE Journal of Solid-State Circuits, vol. 50, no. 7, pp. 1592-1603, July (2015). doi: 10.1109/JSSC.2015.2436875

16. B. Murmann and B. E. Boser, "A 12-bit 75-MS/s pipelined ADC using open-loop residue amplification," in IEEE Journal of Solid-State Circuits, vol. 38, no. 12, pp. 20402050, Dec. (2003). doi: 10.1109/JSSC.2003.819167

17. J. McNeill, M. C. W. Coln and B. J. Larivee, "'Split ADC" architecture for deterministic digital background calibration of a 16-bit 1-MS/s ADC," in IEEE Journal of Solid-State Circuits, vol. 40, no. 12, pp. 2437-2445, Dec. (2005). doi: 10.1109/JSSC.2005.856291

18. L. Dorrer, F. Kuttner, P. Greco, P. Torta and T. Hartig, "A 3-mW 74-dB SNR 2-MHz continuous-time delta-sigma $\mathrm{ADC}$ with a tracking $\mathrm{ADC}$ quantizer in $0.13-/ \mathrm{spl} \mathrm{mu} / \mathrm{m}$

*Hemlata Dalmia, dalmiahemlata@gmail.com
CMOS," in IEEE Journal of Solid-State Circuits, vol. 40, no. 12, pp. 2416-2427, Dec. (2005). doi: 10.1109/JSSC.2005.856282

19. A. Zandieh, P. Schvan and S. P. Voinigescu, "Design of a 55-nm SiGe BiCMOS 5-bit Time-Interleaved Flash ADC for 64-Gbd 16-QAM Fiberoptics Applications," in IEEE Journal of Solid-State Circuits, vol. 54, no. 9, pp. 23752387, Sept. (2019). doi: 10.1109/JSSC.2019.2917155

20. B. Zeinali, T. Moosazadeh, M. Yavari and A. RodriguezVazquez, "Equalization-Based Digital Background Calibration Technique for Pipelined ADCs," in IEEE Transactions on Very Large-Scale Integration (VLSI) Systems, vol. 22, no. 2, pp. 322-333, Feb. (2014). doi: 10.1109/TVLSI.2013.2242208

21. C. Liu, M. Huang and Y. Tu, "A 12 bit 100 MS/s SARAssisted Digital-Slope ADC," in IEEE Journal of SolidState Circuits, vol. 51, no. 12, pp. 2941-2950, Dec. 2016. doi: 10.1109/JSSC.2016.2591822

22. Masumeh Damghanian and Seyed Javad Azhari, A low power 6-bit MOS CML flash ADC with a novel multisegment encoder for UWB applications, Integration, the VLSI Journal, (2017), http://dx.doi.org/10.1016/j.vlsi.2017.01.006

23. A. J. Ginés, E. J. Peralías and A. Rueda, "Black-Box Calibration for ADCs With Hard Nonlinear Errors Using a Novel INL-Based Additive Code: A Pipeline ADC Case Study," in IEEE Transactions on Circuits and Systems I: Regular Papers, vol. 64, no. 7, pp. 1718-1729, (July 2017). doi: 10.1109/TCSI.2017.2662085

24. B. Jeon, S. Hong and O. Kwon, "A Low-Power 12-Bit Extended Counting ADC Without Calibration for CMOS Image Sensors," in IEEE Transactions on Circuits and Systems II: Express Briefs, vol. 65, no. 7, pp. 824-828, (July 2018). doi: 10.1109/TCSII.2017.2717044

25. Kazeminia, S., INTEGRATION the VLSI journal (2017), https://doi.org/10.1016/j.vlsi.2017.11.005

26. M. A. Montazerolghaem, T. Moosazadeh and M. Yavari, "A Single Channel Split ADC Structure for Digital Background Calibration in Pipelined ADCs," in IEEE Transactions on Very Large-Scale Integration (VLSI) Systems, vol. 25, no. 4, pp. 1563-1567, April (2017). doi: 10.1109/TVLSI.2016.2641259

27. S. Kazeminia, A real-time pseudo-background gain calibration strategy for residue amplifiers of pipeline ADCs, Integration, the VLSI Journal, (2018), https://doi.org/10.1016/j.vlsi.2018.11.003

28. K. Muroya et al., "900-MHz, 3.5-mW, 8-bit pipelined subranging ADC combining flash ADC and TDC," 2017 IEEE International Symposium on Radio-Frequency Integration Technology (RFIT), Seoul, (2017), pp. 7-9. doi: 10.1109/RFIT.2017.8048272

29. A. Wang, C.-J.R. Shi, A 10-bit 50-MS/s SAR ADC with 1 $\mathrm{fJ} /$ Conversion in $14 \mathrm{~nm}$ SOI FinFET CMOS, Integration, the VLSI Journal (2018), https://doi.org/10.1016/j.vlsi.2018.03.010

30. K. Yoshioka et al., "A 20-ch TDC/ADC Hybrid Architecture LiDAR SoC for 240 \$ \times\$ 96 Pixel 200-m Range Imaging with Smart Accumulation Technique and 
Residue Quantizing SAR ADC," in IEEE Journal of SolidState Circuits, vol. 53, no. 11, pp. 3026-3038, Nov. (2018). doi: 10.1109/JSSC.2018.2868315

31. A Review on an Efficient Architecture of Pipeline ADC for High Speed Applications, KB Vaibhav "A Meshram, International Journal of Engineering Research and Applications", July (2019), pp 01-08, DOI: 10.9790/96220907060108

32. M. Savitha,R. Venkat Siva Reddy, "Dual split-three segment capacitor array Design Based Successive approximation ADC for Io-T ecosystem", Integration, the VLSI Journal, Elsevier, November (2019)

33. T. Hung, F. Liao and T. Kuo, "A 12-Bit Time-Interleaved 400-MS/s Pipelined ADC With Split-ADC Digital Background Calibration in 4,000 Conversions/Channel," in IEEE Transactions on Circuits and Systems II: Express Briefs, vol. 66, no. 11, pp. 1810-1814, Nov. (2019).

34. M. H. Naderi, C. Park, S. Prakash, M. Kinyua, E. G. Soenen and J. Silva-Martinez, "A 27.7 fJ/conv-step 500 MS/s 12-Bit Pipelined ADC Employing a Sub-ADC Forecasting Technique and Low-Power Class AB Slew Boosted Amplifiers," in IEEE Transactions on Circuits and Systems I: Regular Papers, vol. 66, no. 9, pp. 3352-3364, Sept. (2019).

35. A. Mahmoudi, et al., A study of analog decision feedback equalization for ADC-Based serial link receivers, Integration, the VLSI Journal (2018), https://doi.org/10.1016/j.vlsi.2018.09.003

36. Y. Zhou, B. Xu and Y. Chiu, "A 12-b 1-GS/s 31.5-mW Time-Interleaved SAR ADC with Analog HPF-Assisted Skew Calibration and Randomly Sampling Reference ADC," in IEEE Journal of Solid-State Circuits, vol. 54, no. 8, pp. 2207-2218, Aug. (2019).

37. J. Xu, P. Harpe and C. Van Hoof, "An Energy-Efficient and Reconfigurable Sensor IC for Bio-Impedance Spectroscopy and ECG Recording," in IEEE Journal on Emerging and Selected Topics in Circuits and Systems, vol. 8, no. 3, pp. 616-626, Sept. (2018).

38. K. Muroya et al., "900-MHz, 3.5-mW, 8-bit pipelined subranging ADC combining flash ADC and TDC," 2017 IEEE International Symposium on Radio-Frequency Integration Technology (RFIT), Seoul, (2017), pp. 7-9.

39. H. Fan et al., "High-resolution ADCs design in sensors," 2018 IEEE 9th Latin American Symposium on Circuits \& Systems (LASCAS), Puerto Vallarta, (2018), pp. 1-4.

40. C. Chen et al., "A Pitch-Matched Front-End ASIC With Integrated Subarray Beamforming ADC for Miniature 3-D Ultrasound Probes," in IEEE Journal of Solid-State Circuits, vol. 53, no. 11, pp. 3050-3064, Nov. (2018).

41. A. Gupta, A. Singh and A. Agarwal, "Highly-digital voltage scalable 4-bit flash ADC," in IET Circuits, Devices \& Systems, vol. 13, no. 1, pp. 91-97, 1 (2019).

42. Bankupalli, P.T., Srikanth Babu, V., Suresh Kumar. T, "Modelling of static VAR compensator employing a cascaded H-bridged multilevel converter", International Journal of Applied Engineering Research, vol. 10, no. 16, pp 37057-37062

*Hemlata Dalmia, dalmiahemlata@gmail.com
43. A. A. Abualsaud, S. Qaisar, S. H. Ba-Abdullah, Z. M. AlSheikh and M. Akbar, "Design and implementation of a 5bit flash ADC for education," 2016 5th International Conference on Electronic Devices, Systems and Applications (ICEDSA), Ras Al Khaimah, (2016), pp. 1-4.

44. Ginés AJ, Peralías EJ, Aledo C, Rueda A. Fast adaptive comparator offset calibration in pipeline ADC with self-repairing thermometer to binary encoder. Int J Circ Theor Appl. (2019) ;1-17. https://doi. org/10.1002/cta.2594

45. Xiangyu Liu1, Hui Xu, Yinan Wang, Yingqiang Dai, Nan $\mathrm{Li}$, and Guiqing Liu, "Calibration for Sample-and-Hold Mismatches in M-Channel TI ADCs Based on Statistics", in Applied Sciences (MDPI publisher), Jan (2019) doi: 10.3390/app9010198,

46. Jingyu Li, Jiameng Pan and Yue Zhang, "Automatic Calibration Method of Channel Mismatches for Wideband TI-ADC System", in Electronics (MDPI publisher), (2019), 8, 56; doi:10.3390/electronics 8010056

47. J Monica and P.Kannan, "A REVIEW ON DESIGN OF PIPELINED ADC", in International Journal of Advanced Research in Computer Engineering \& Technology (IJARCET) Volume 3 Issue 11, November (2014)

48. A.S. Korotkov, Methods of calibration and correction of analog-to-digital converters (review). Russ Microelectron 43, 226-237

(2014). https://doi.org/10.1134/S1063739714030044

49. Xin Li, Cheng Huang, Desheng Ding and Jianhui Wu, A Review on Calibration Methods of Timing-skew in Timeinterleaved ADCs, Journal of Circuits, Systems and Computers, March (2019), doi: 10.1142/S0218126620300020

50. Seyed Alireza Zahrai and Marvin Onabajo, "Review of Analog-To-Digital Conversion Characteristics and Design Considerations for the Creation of Power-Efficient Hybrid Data Converters", in Journal of Low Power Electronics and Applications, (2018), 8, 12; doi:10.3390/jlpea8020012

51. B. $\mathrm{Xu}$ and Y. Chiu, "Comprehensive Background Calibration of Time-Interleaved Analog-to-Digital Converters," in IEEE Transactions on Circuits and Systems I: Regular Papers, vol. 62, no. 5, pp. 1306-1314, May (2015).

52. Silpa Kesav, K.S.Nayanathara and B.K. Madhavi, "DESIGN OF LOW POWER SAR ADC FOR ECG USING 45nm CMOS TECHNOLOGY" in International Journal of VLSI design \& Communication Systems (VLSICS) Vol.8, No.1, February (2017) 10.1109/RFIT.2017.8048272

53. E. Santin, L. B. Oliveira, B. Nowacki and J. Goes, "A Fully Integrated and Reconfigurable Architecture for Coherent Self-Testing of High-Speed Analog-to-Digital Converters," in IEEE Transactions on Circuits and Systems I: Regular Papers, vol. 58, no. 7, pp. 1531-1541, July (2011).

54. P. Ramakrishna, K. Hari Kishore, "Design of Low Power 10GS/s 6-Bit DAC using CMOS Technology" International Journal of Engineering and Technology Volume-7, Issue-2, Jan - (2018).

55. Frank M. Yaul, Anantha P. Chandrakasan “A 10b 0.6nW SAR ADC with Data-Dependent Energy Savings Using LSB-First Successive Approximation" IEEE International Solid-State Circuits Conference (2014) 
56. Sunghyuk Lee, Anantha P. Chandrakasan, Hae-Seung Lee, "A $1 \mathrm{GS} / \mathrm{s}$ 10b $18.9 \mathrm{~mW}$ Time- Interleaved SAR ADC With Background Timing Skew Calibration" IEEE JOURNAL OF SOLIDSTATE CIRCUITS, VOL. 49, NO. 12, DECEMBER (2014)

57. Ahmed M. A. Ali, Christopher Dillon, Robert Sneed, Andrew S. Morgan, Scott Bardsley, John Kornblum, and $\mathrm{Lu} \mathrm{Wu} \mathrm{"A} \mathrm{14-bit} 125 \mathrm{MS} / \mathrm{s}$ IF/RF Sampling Pipelined ADC With $100 \mathrm{~dB}$ SFDR and 50 fs Jitter" IEEE JOURNAL OF SOLID-STATE CIRCUITS, VOL. 41, NO. 8, AUGUST (2006)

58. Yunzhi Dong, William Yang, Richard Schreier, Ali Sheikholeslami, Sudhir Korrapati "A Continuous-Time 03 MASH ADC Achieving $88 \mathrm{~dB}$ DR With $53 \mathrm{MHz}$ BW in 28 nm CMOS" IEEE JOURNAL OF SOLID-STATE CIRCUITS, VOL. 49, NO. 12, DECEMBER (2014)

59. Daniel R. McMahill, Dwaine S. Hurta, Brian Brandt, Miaochen Wu, Paul Kalthoff, Geir S. Ostrem, "A 160 Channel QAM Modulator With 4.6 Gsps 14 Bit DAC" IEEE JOURNAL OF SOLID-STATE CIRCUITS, VOL. 49, NO. 12, DECEMBER (2014)

60. Luca Bettini, Thomas Christen, Thomas Burger and Qiuting Huang "A Reconfigurable DT Sigma Modulator for Multi-Standard 2G/3G/4G Wireless Receivers" IEEE JOURNAL ON EMERGING AND SELECTED TOPICS IN CIRCUITS AND SYSTEMS, 2156-3357, September $18,(2015)$

61. Yang Xu, Zehong Zhang, Baoyong Chi, Nan Qi, Hualin Cai and Zhihua Wang "A 5-/20-MHz BW Reconfigurable Quadrature Bandpass CT Sigma ADC With Anti-PoleSplitting Opamp and Digital I /Q Calibration" IEEE TRANSACTIONS ON VERY LARGE-SCALE INTEGRATION (VLSI) SYSTEMS 1063-8210 C (2015) IEEE.

62. SayfeKiaei, Eby G. Friedman "Introduction to the Special Issue on Low Power Wireless Communications" IEEE TRANSACTIONS ON CIRCUITS AND SYSTEMS-II: ANALOG AND DIGITAL SIGNAL PROCESSING, VOL. 44, NO. 6, JUNE (1997)

63. T. Christen and Q. Huang, "A 0.13 CMOS 0.1-20 MHz bandwidth 86-70 dB DR multi-mode DT ADC for IMTadvanced," in Proc.IEEE Eur. Solid-State Circuits Conf., Sep. (2010), pp. 414- 417.

64. Terence C. Randall, Ifana Mahbub, Syed K. Islam "Reconfigurable Analog-to-Digital Converter for Implantable Bioimpedance Monitoring" BioWireleSS (2015),78-1-4799-5511-4/15@2015-IEEE

65. Huailiang $\mathrm{Li}$ and Jing $\mathrm{Hu}$, The Research on SAR ADC Integrated Circuit (2019), doi:10.1088/17426596/1314/1/012022

66. J. -. Park, D. -. Kim, T. -. An, M. -. Kim, G. -. Ahn and S. . Lee, "12 b $50 \mathrm{MS} / \mathrm{s} 0.18 \mu \mathrm{m}$ CMOS SAR ADC based on highly linear C-R hybrid DAC," in Electronics Letters, vol. 56, no. 3, pp. 119-121, 62 (2020)

67. B. D. Kumar, H. Shrimali and N. Gupta, "A 6-Bit, 29.56 fJ/Conv-Step, Voltage Scalable Flash-SAR Hybrid ADC in 28 nm CMOS," 2019 IEEE International Symposium on
Circuits and Systems (ISCAS), Sapporo, Japan, (2019), pp. $1-5$.

68. T. Lee, B. Verbruggen and U. Moon, "Session 28 overview: Hybrid ADCs," 2017 IEEE International SolidState Circuits Conference (ISSCC), San Francisco, CA, (2017), pp. 464-465.

69. K. Yoshioka et al., "Digital Amplifier: A Power-Efficient and Process-Scaling Amplifier for Switched Capacitor Circuits," in IEEE Transactions on Very Large-Scale Integration (VLSI) Systems, vol. 27, no. 11, pp. 25752586, Nov. (2019).

70. K. Yoshioka, A. Shikata, R. Sekimoto, T. Kuroda and H. Ishikuro, "An 8 bit 0.3-0.8 V 0.2-40 MS/s 2-bit/Step SAR ADC With Successively Activated Threshold Configuring Comparators in $40 \mathrm{~nm}$ CMOS," in IEEE Transactions on Very Large Scale Integration (VLSI) Systems, vol. 23, no. 2, pp. 356-368, Feb. (2015).

71. K. Yoshioka, R. Saito, T. Danjo, S. Tsukamoto and H. Ishikuro, "Dynamic Architecture and Frequency Scaling in 0.8-1.2 GS/s $7 \mathrm{~b}$ Subranging ADC," in IEEE Journal of Solid-State Circuits, vol. 50, no. 4, pp. 932-945, (April 2015).

72. Yoshioka, K., Sugimoto, T., Waki, N., Kim, S., Kurose, D., Ishii, H., ... \& Itakura, T, "28.7 A 0.7 V 12b 160MS/s $12.8 \mathrm{fJ} /$ conv-step pipelined-SAR ADC in $28 \mathrm{~nm}$ CMOS with digital amplifier technique. In 2017 IEEE International Solid-State Circuits Conference (ISSCC) (pp. 478-479). IEEE.

73. Yoshioka, Kentaro, Ryo Saito, Takumi Danjo, Sanroku Tsukamoto, and Hiroki Ishikuro. "7-bit $0.8-1.2 \mathrm{GS} / \mathrm{s}$ dynamic architecture and frequency scaling subrange ADC with binary-search/flash live configuring technique." In 2014 Symposium on VLSI Circuits Digest of Technical Papers, pp. 1-2. IEEE, (2014).

74. K. Yoshioka, A. Shikata, R. Sekimoto, T. Kuroda and H. Ishikuro, "An 8 bit $0.3-0.8 \mathrm{~V}$ 0.2-40 MS/s 2-bit/Step SAR ADC With Successively Activated Threshold Configuring Comparators in $40 \mathrm{~nm}$ CMOS," in IEEE Transactions on Very Large Scale Integration (VLSI) Systems, vol. 23, no. 2, pp. 356-368, Feb. (2015).

75. K. Yoshioka, A. Shikata, R. Sekimoto, T. Kuroda and H. Ishikuro, "A $0.0058 \mathrm{~mm} 27.0$ ENOB $24 \mathrm{MS} / \mathrm{s} 17 \mathrm{fJ} / \mathrm{conv}$. threshold configuring SAR ADC with source voltage shifting and interpolation technique," (2013) Symposium on VLSI Circuits, Kyoto, 2013, pp. C266-C267.

76. Yoshioka, Kentaro, Akira Shikata, Ryota Sekimoto, Tadahiro Kuroda, and Hiroki Ishikuro. "A 0.35-0.8 V 8b $0.5-35 \mathrm{MS} / \mathrm{s} 2 \mathrm{bit} / \mathrm{step}$ extremely-low power SAR ADC." In 2013 18th Asia and South Pacific Design Automation Conference (ASP-DAC), pp. 111-112. IEEE, (2013).

77. Yoshioka, Kentaro, Akira Shikata, Ryota Sekimoto, Tadahiro Kuroda, and Hiroki Ishikuro. "An 8bit 0.35-0.8 $\mathrm{V} 0.5-30 \mathrm{MS} / \mathrm{s}$ 2bit/step SAR ADC with wide range threshold configuring comparator." In 2012 Proceedings of the ESSCIRC (ESSCIRC), pp. 381-384. IEEE, (2012).

78. B. Murmann, "ADC Performance Survey 1997-2020," [Online]. Available: http://web.stanford.edu/ murmann/adcsurvey.html. 
79. Zahrai, S. A., Zlochisti, M., Le Dortz, N., \& Onabajo, M. (2017). A low-power high-speed hybrid ADC with merged sample-and-hold and DAC functions for efficient subranging time-interleaved operation. IEEE Transactions on Very Large-Scale Integration (VLSI) Systems, 25(11), 3193-3206.

80. S. Kim and K. Kwon, "A hybrid ADC combining capacitive DAC-based multi-bit/cycle SAR ADC with flash ADC," 2016 International Conference on Electronics, Information, and Communications (ICEIC), Da Nang, (2016), pp. 1-4.

81. Gönen, Burak, Fabio Sebastiano, Robert van Veldhoven, and Kofi AA Makinwa. "A hybrid ADC for high resolution: The zoom ADC." In Hybrid ADCs, Smart Sensors for the IoT, and Sub-1V \& Advanced Node Analog Circuit Design, pp. 99-117. Springer, Cham, (2018).

82. Molaei, Hasan, Khosrow Hajsadeghi, and Ata Khorami. "Design of low power comparator-reduced hybrid ADC." Microelectronics Journal 79 (2018): 79-90.

83. Kim, Min-Kyu, Min-Seok Shin, Yun-Rae Jo, Jong-Boo Kim, Jaseung Gou, Sangdong Yoo, and Oh-Kyong Kwon. "A $\Delta \Sigma$-cyclic hybrid ADC for parallel readout sensor applications." In 2012 IEEE International Symposium on Circuits and Systems, pp. 532-535. IEEE, (2012).

84. Jianwen Li 1,2, Xuan Guo 1, *, Jian Luan 1,2, DanyuWu 1, Lei Zhou 1, NanxunWu 2, Yinkun Huang 1, Hanbo Jia 1,2, Xuqiang Zheng 1, JinWu 1 and Xinyu Liu, "A 1 GS/s 12Bit Pipelined/SAR Hybrid ADC in $40 \mathrm{~nm}$ CMOS Technology" in Electronics (MDPI), Feb (2020)

85. Razzaq, Anas, and Shabbir Majeed Chaudhry. "A 15-Bit $85 \mathrm{MS} / \mathrm{s}$ Hybrid Flash-SAR ADC in 90-nm CMOS." Circuits, Systems, and Signal Processing 37.4 (2018): 1452-1478.

86. Huang, Zhaofeng, et al. "A 16-bit Hybrid ADC with Circular-Adder-Based Counting for $15 \mu \mathrm{m}$ Pitch $640 \times 512$ LWIR FPAs." Chinese Journal of Electronics 29.2 (2020): 291-296.

87. Lee, Hsun-Cheng, and Jacob A. Abraham. "A novel low power 11-bit hybrid ADC using flash and delay line architectures." 2014 Design, Automation \& Test in Europe Conference \& Exhibition (DATE). IEEE, (2014).

88. Dutt, Samir. "A novel 10-bit hybrid ADC using flash and delay line architectures." PhD diss., (2011).

89. C. Lee and M. P. Flynn, "A SAR Assisted 2-Stage Pipeline ADC," IEEE Journal of Solid-State Circuits, (April 2011)

90. C. Lee and M. P. Flynn, "A 12b 50MS/s 3.5mW SAR Assisted 2-Stage Pipeline ADC," IEEE Symposium on VLSI Circuits, (June 2010)

91. Brandolini, M., Shin, Y., Raviprakash, K., Wang, T., Wu, R., Geddada, H. M., ... \& Hsieh, M. H. (2015, February). $26.6 \mathrm{a} 5 \mathrm{gs} / \mathrm{s} 150 \mathrm{mw} 10 \mathrm{~b}$ sha-less pipelined/sar hybrid adc in $28 \mathrm{~nm}$ cmos. In (2015) IEEE International Solid-State Circuits Conference-(ISSCC) Digest of Technical Papers (pp. 1-3). IEEE.

92. Mo, Jianhua, et al. "Hybrid architectures with few-bit ADC receivers: Achievable rates and energy-rate tradeoffs."

*Hemlata Dalmia, dalmiahemlata@gmail.com
IEEE Transactions on Wireless Communications 16.4 (2017): 2274-2287.

93. Fang, Xiang, et al. "CMOS 12 bits 50kS/s micropower SAR and dual-slope hybrid ADC." 2009 52nd IEEE International Midwest Symposium on Circuits and Systems. IEEE, (2009).

94. Shafik, Ayman, et al. "3.6 A 10Gb/s hybrid ADC-based receiver with embedded 3-tap analog FFE and dynamically-enabled digital equalization in 65nm CMOS." 2015 IEEE International Solid-State Circuits Conference(ISSCC) Digest of Technical Papers. IEEE, (2015). 